\title{
Effect of porous partition height on thermal performance of a ventilated cavity using LBM- MRT
}

\author{
Zouhira Hireche ${ }^{l}$, Nabil Himrane ${ }^{2}$, Lyes Nasseri ${ }^{1 *}$, and Djamel Eddine Ameziani ${ }^{1}$ \\ ${ }^{1}$ LTPMP Laboratory. Faculty of Mechanical and Proceeding Engineering, University of Sciences and \\ Technology Houari Boumediene, Algiers, Algeria; \\ ${ }^{2}$ Labo of Energy and Mechanical Engineering (LEMI), Faculty of Technology, UMBB, Boumerdes, \\ Algeria
}

\begin{abstract}
The objective of this work is to study the effect of the thickness of a porous separation on the thermal performance in a cavity with displacement ventilation. The cold air jet enters and exits through two openings located in the lower and upper parts of the left wall and the right wall respectively. The other horizontal walls are also adiabatic. The hydrodynamic and thermal characteristics of the transfer were studied for three configurations with the same aspect ratio $\mathrm{L} / \mathrm{H}=2$. The height $\mathrm{Hp}$ of the porous separation was varied between 0.2 and 0.8 where is placed in the center of the cavity. The transfer rates on the active wall for the thicknesses were studied for different permeability therefore different Darcy numbers varying over an interval: $10^{-6} \leq \mathrm{Da} \leq 10$. The dimensionless Rayleigh and Reynolds numbers were taken from the rows: $10 \leq \mathrm{Ra} \leq 10^{6}$ and $50 \leq \mathrm{Re} \leq 500$. The governing equations of momentum and energy were solved by the Lettice Boltzmann Multiple Relaxation Time Method (LB-MRT) D2Q9 for the velocity field and D2Q5 for the temperature field. In order to take into account the introduction of the porous medium, an additional term is added to the standard LB equations based on the generalized model (Darcy model extended to Brinkman-Forchheimer).
\end{abstract}

\section{Introduction}

The study of displacement ventilation (DV) in rectangular enclosure is of interest in a wide range of civil and industrial applications [1], such as chemical applications, thermal and pollution control [2,3], and building applications [4-7].

The all-important challenge in modern building design for ventilation is to provide thermal comfort and better indoor air quality, since, many people pass most of their time into commercial or residential buildings. Ventilation is attested to be the most appropriate way to ensure a good thermal comfort (heating/cooling). To ventilate a space, two techniques are involved: one is buoyancy driven flow and others is mechanical driven flow (fresh air). The combination of these two modes introduces the mixed convection problem inside the

\footnotetext{
* Corresponding author:lyes.nasseri@gmil.com
} 
ventilated space. So, mixed convection is that (mode of heat exchange) the heat transfer mode in which there is a main interaction between natural convection and forced convection. Principally, mixing ventilation (MV) and displacement ventilation (DV) are the most two popular air distribution flow applied to recirculate the indoor environment. In mixing ventilation, fresh air is introduced at a high velocity from the ceiling level to attain a uniform distribution of temperature and concentration in the room [8-10], or by mixing air with internal ventilation $[11,12]$. However, on account of the high velocity, mixed ventilation is still lacking comfort in workplaces and effective in pollutant removal [13]. For all ventilation systems, the locations of return air outlets have a great effect on his performance [14]. Note that in displacement ventilation air supply diffusers are located near floor level and the outdoor air velocity is very low compared with the first mode (i.e. MV), and exhausted air is extracted from the ceiling level by buoyancy forces. And the locations of return air outlets have a great effect on the performance of the ventilation system.

Several publications have appeared in recent years comparing fluid flows between mixed ventilation and displacement ventilation. Ren et al. [15] studied flow mixing and displacement flow inside a slot vented enclosure in the presence of heated and airborne polluted strip. Heat and mass transfers are visualized for both ventilation mode, and their efficiency are compared. Also, a numerical investigation due to heated and polluted strip inside an open cavity in the presence of conducting baffle was presented by Ren et al. [16] to optimize the removal rate of heat and pollutants using heat lines and mass lines. They found that are mostly affected by the length and of the position baffle. Also, the heat and mass transfer rate can be increased by the baffle only when its distance from the bottom wall varies between 0.2 and 0.5 with thermal Rayleigh number higher than a critical value.

As seen, the review of the literature indicates that the displacement ventilation heat transfer in enclosure has been addressed in many of previous research; however, there are only few researches which have considered porous obstacles inside the cavity, and no studies do undertake into account a vertical porous partition. Altogether, this model could represent the process of heat removal by displacement ventilation in rectangular enclosure such as: large room, open space office, or general ward of hospital.

\section{Mathematical formulation and numerical resolution}

The present study concerns with mixed convection in a rectangular cavity with side length and height $\mathrm{L}$ and $\mathrm{H}$ as shown in fig. 1. Air in the cavity is renewed through an input and output ports, located at the lower and upper ends part of the left and right walls, respectively, with an opening value of $\mathrm{H} / 10$. A separation porous wall saturated with the same fluid (e.g. air), is placed in the middle of the bottom surface whose thickness is $\mathrm{Ep}=\mathrm{H} / 5$ and height between $\mathrm{H} / 5$ to $4 \mathrm{H} / 5$. Cavity walls are impermeable, so the left wall is maintained at a hot temperature $\mathrm{T}_{\mathrm{h}}$, and the others wall are thermally insulated. 


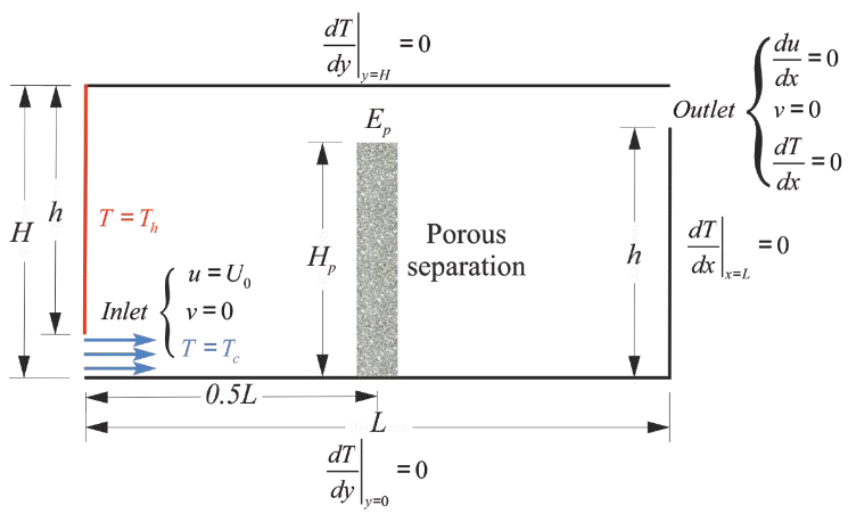

Fig. 1.Physical model

Based on the generalized model Darcy-Brinkman-Forchheimer, the dimensionless governing equations for convection heat transfer in a homogeneous, isotropic and fluid-saturated porous medium can be written as [17-18]:

Continuity equation:

$$
\frac{\partial(U)}{\partial X}+\frac{\partial(V)}{\partial Y}=0
$$

$\mathrm{X}$ Momentum conservation equation:

$$
\frac{\partial U}{\partial t}+\left[U \frac{\partial}{\partial X}\left(\frac{U}{\varepsilon}\right)+V \frac{\partial}{\partial Y}\left(\frac{U}{\varepsilon}\right)\right]=-\frac{\partial \varepsilon P}{\partial X}+\frac{1}{\operatorname{Re}}\left(\frac{\partial^{2} U}{\partial X^{2}}+\frac{\partial^{2} U}{\partial Y^{2}}\right)+G r_{1}
$$

Y Momentum conservation equation:

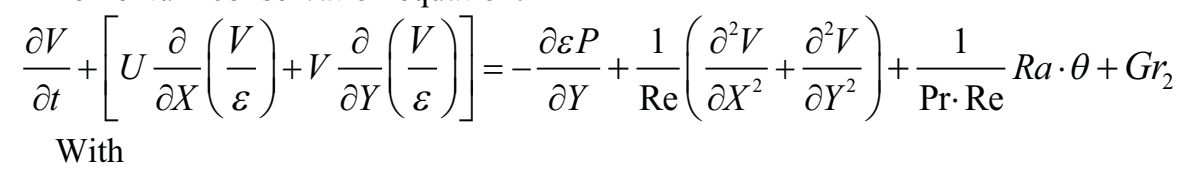

$G r_{1}=-\frac{1}{D a \operatorname{Re}} U-\frac{1.75}{\sqrt{150}} \frac{\left(U^{2}+V^{2}\right)^{1 / 2}}{\sqrt{D a}} U$ and $G r_{2}=-\frac{1}{D a \operatorname{Re}} V-\frac{1.75}{\sqrt{150}} \frac{\left(U^{2}+V^{2}\right)^{1 / 2}}{\sqrt{D a}} V$

Energy conservation equation:

$$
\frac{\partial \theta}{\partial t}+\frac{\partial(U \theta)}{\partial X}+\frac{\partial(V \theta)}{\partial Y}=\frac{1}{\operatorname{Re} \operatorname{Pr}}\left(\frac{\partial^{2} \theta}{\partial X^{2}}+\frac{\partial^{2} \theta}{\partial Y^{2}}\right)
$$

The control parameters appeared in the generalized equations are the following nondimensional numbers:

$$
\operatorname{Pr}=\frac{v}{\alpha}, \operatorname{Re}=\frac{U_{0} H}{v}, R a=\frac{g \beta \Delta T_{r e f} H^{3}}{v \alpha}, D a=\frac{K}{H^{2}}
$$

Respectively Prandtl, Reynolds, Rayleigh and Darcy numbers. The non-dimensional variables used in the construction of the mathematical model are:

$$
X=\frac{x}{H}, Y=\frac{y}{H}, U=\frac{u}{U_{0}}, V=\frac{v}{U_{0}}, P=\frac{p}{\rho U_{0}^{2}}, \theta=\frac{T(t)-T_{c}}{\Delta T_{r e f}}, \Delta T_{r e f}=T_{h}-T_{c},
$$

Hydrodynamic boundary conditions: characterized by the non-slip condition of the fluid particles, on the solid walls and their impermeability are given: 
Left vertical wall:

$$
U(0, Y)=V(0, Y)=0 ; \quad 0.1 \leq Y \leq 1
$$

Right vertical wall:

$$
U(2, Y)=V(2, Y)=0 ; \quad 0 \leq Y \leq 0.9
$$

Horizontal surfaces: $0 \leq X \leq 2$

$$
U(X, 0)=V(X, 0)=U(X, 1)=V(X, 1)=0 ;
$$

The inlet:

$$
\left\{\begin{array}{l}
X=0 \\
0 \leq Y \leq 0.1
\end{array} \quad U=1, \quad V=0\right.
$$

The outlet:

$$
\left\{\begin{array}{l}
X=2 \\
0.9 \leq Y \leq 1
\end{array} \quad \frac{\partial U}{\partial X}=0, \quad V=0 .\right.
$$

Thermal boundary conditions:

Left vertical wall:

$$
\theta=1, \quad X=0,0.1 \leq Y \leq 1
$$

The inlet:

$$
\theta=0, \quad 0 \leq Y \leq 0.1
$$

Right vertical wall:

$$
\left.\frac{\partial \theta}{\partial X}\right|_{X=2}=0, \quad 0 \leq Y \leq 0.9
$$

The outlet:

$$
\left.\frac{\partial \theta}{\partial X}\right|_{X=2}=0, \quad 0.9 \leq Y \leq 1
$$

Horizontal surfaces:

$$
\left.\frac{\partial \theta}{\partial Y}\right|_{Y=0}=0,\left.\frac{\partial \theta}{\partial Y}\right|_{Y=1}=0 ; \quad 0 \leq X \leq 2
$$

The heat transfer rate at the hot wall is obtained after the establishment of physical properties (steady state) and it is defined as follows:

$$
N u=\int_{0.1}^{1} N u_{X} d Y \quad \text { with } \quad N u_{X}=\left.\frac{\partial \theta}{\partial X}\right|_{X=0}
$$

The Lattice Boltzmann method with multi-relaxation times "LBM-MRT" which will be used to solve the governing equations of our problem.

\subsection{Double LB-MRT model for thermal flow in porous media}

The evolution equation of the Multiple Relaxation Time Lattice Boltzmann model for porous flow is:

$$
f_{i}\left(r_{j}+c_{i} \Delta t, t_{k}+\Delta t\right)=f_{i}\left(r_{j}, t_{k}\right)-M^{-1} S\left[m\left(r_{j}, t_{k}\right)-m^{e q}\left(r_{j}, t_{k}\right)\right]+\Delta t M^{-1}\left(1-\frac{S}{2}\right) F(18)
$$


where $f(r, t)$ is the 9-dimensional vector of the distribution functions $f$ at the node $r$ and time $t ; \Delta T$ the time step, $S$ the collision operator given by $S=\operatorname{diag}\left(S_{0}, S_{1}, S_{2}, S_{3}, S_{4}, S_{5}, S_{6}, S_{7}, S_{8}\right) . c_{i}$ is the discrete velocity vector specified by the $D 2 Q 9$ discrete velocities set, and $m=M f$ is the moment vector, $m^{e q}$ the equilibria in moment space. $F$ denotes the forcing term in the moment space, and $M$ is a non-orthogonal transformation matrix given by [19].

Using the transformation matrix, the moments can be constructed by mapping the discrete distribution functions $f$ in the velocity space onto the moment space, which gives:

$$
m=M f=\left(\rho, j_{x}, j_{y}, E, \bar{\varepsilon}, \varphi_{x}, \varphi_{y}, p_{x x}, p_{y y}\right)^{T}
$$

where $\rho$ is the density, $E$ is the total energy, $\bar{\varepsilon}$ is related to the energy, $j_{x}$ and $j_{y}$ are the components of the momentum, $\varphi_{x}$ and $\varphi_{y}$ are related to the energy flux, and $p_{x x}$ and $p_{y y}$ are related to the strain tensor, respectively. And similarly, the corresponding equilibria in moment space are given by:

$$
\begin{aligned}
& m^{e q}=M f^{e q}=\left(\rho, j_{x}, j_{y}, E^{e q}, \bar{\varepsilon}^{e q}, \varphi_{x}^{e q}, \varphi_{y}^{e q}, p_{x x}, p_{y y}\right)^{T} \\
= & \rho\left(1, u_{x}, u_{y}, \frac{2}{3}+\frac{u^{2}}{\varepsilon}, \frac{u_{x}^{2}-u_{y}^{2}}{\varepsilon}, \frac{u_{x} u_{y}}{\varepsilon}, \frac{1}{3} u_{y}, \frac{1}{3} u_{x}, \frac{1}{9}+\frac{1}{3} \frac{u^{2}}{\varepsilon}\right)^{T}
\end{aligned}
$$

where:

$$
\begin{gathered}
f=\left(f_{0}, f_{1}, \ldots, f_{8}\right)^{T}, f^{e q}=\left(f_{0}^{e q}, f_{1}^{e q}, \ldots, f_{8}^{e q}\right)^{T} \\
\text { and } u=\left(u_{x}, u_{y}\right)
\end{gathered}
$$

The equilibrium distribution function is given by:

$$
f_{i}^{e q}=\omega_{i} \rho\left[1+\frac{\vec{c}_{i} \cdot \vec{u}}{c_{s}{ }^{2}}+\frac{\left(\vec{c}_{i} \cdot \vec{u}\right)^{2}}{2 \varepsilon c_{s}{ }^{4}}-\frac{\vec{u} \cdot \vec{u}}{2 \varepsilon c_{s}{ }^{2}}\right] ; \text { for } i=0,8
$$

where $c_{s}=\sqrt{R T}=c / \sqrt{3}$ is the sound speed of the D2Q9 model, in which $R$ is the gas constant, $T$ is the temperature, and $c=\delta x / \delta t, \delta x=\delta t=1$ with $\delta x$ the discrete lattice spacing.

$\omega_{i}$ is the weight coefficient given by: $\omega_{0}=\frac{4}{9}, \omega_{i}=1 / 9$ for $i=1,2,3,4$, $\omega_{i}=1 / 36$ for $i=5,6,7,8$.

The forcing term $F$ in the moment space is given by:

$$
\begin{aligned}
& F_{0}=0, F_{1}=\rho F_{x}, F_{2}=\rho F_{y}, F_{3}=\rho\left(2 u_{x} F_{x}+2 u_{y} F_{y}\right) / \varepsilon, \\
& F_{4}=\rho\left(2 u_{x} F_{x}-2 u_{y} F_{y}\right) / \varepsilon, F_{5}=\rho\left(u_{x} F_{y}+u_{y} F_{x}\right) / \varepsilon, \\
& F_{6}=\frac{1}{3} \rho F_{y}, F_{7}=\frac{1}{3} \rho F_{x}, F_{8}=\frac{2}{3} \rho\left(u_{x} F_{y}+u_{y} F_{x}\right) / \varepsilon,
\end{aligned}
$$

where:

$$
F_{x}=a_{x} \text { and } F_{y}=a_{y}+c_{s}{ }^{2}\left[1+2\left(\tau_{f}-0.5\right) u_{y}\right]
$$


The volume-averaged density $\rho$, velocity $u$ and effective viscosity $v_{e}$ are respectively given by:

$$
\begin{gathered}
\rho=\sum_{i=0}^{8} f_{i} \\
u=v /\left(d_{0}+\sqrt{d_{0}^{2}+d_{1}|v|}\right) \\
v_{e}=c_{s}^{2}\left(\tau_{f}-0.5\right) \Delta T
\end{gathered}
$$

Where $\tau_{f}=\frac{1}{s_{4}}=\frac{1}{s_{5}}, d_{0}=\frac{1}{2}\left[\frac{1+\left(\tau_{f}-0.5\right) R \cdot T \cdot \delta t}{r}+r \varepsilon \frac{\delta t}{2} \frac{v}{K}\right], d_{1}=r \varepsilon \frac{\delta t}{2} \frac{F_{\varepsilon}}{K}$ and $v$ is a temporal velocity defined by:

$$
\nu=\sum_{i=0}^{8} e_{i} f_{i} / \rho+\frac{\delta t}{2} r \varepsilon G+\frac{\delta t}{2} R T \delta_{\alpha}
$$

Through the Chapman-Enskog analysis, it can be proved that the hydrodynamic equations [20] can be recovered by the present MRT LB model for the velocity field.

\subsection{LB-MRT model for temperature field}

To keep consistent with the MRT LB model for the velocity field, the MRT LB model is proposed as:

$$
g_{i}\left(r_{j}+c_{i} \Delta t, t_{k}+\Delta t\right)=g_{i}\left(r_{j}, t_{k}\right)-N^{-1} S\left[n\left(r_{j}, t_{k}\right)-n^{e q}\left(r_{j}, t_{k}\right)\right]+\Delta t N^{-1}\left(\Psi+\left(1-\frac{\Theta}{2}\right) \bar{H}\right)
$$

where $g(r, t)$ is the temperature distribution function. $n(r, t)$ and $n^{e q}(x, t)$ are the moments and equilibrium moments corresponding to $g(r, t)$.

$\left\{e_{i} \mid i=0,1, \ldots, 4\right\}$ is the discrete velocities set specified by the $D 2 Q 5$ model. $\Theta=\operatorname{diag}\left(\sigma_{0}, \sigma_{1}, \sigma_{2}, \sigma_{3}, \sigma_{4}\right)$ is the diagonal relaxation matrix. $N$ is the transformation matrix given by $[11,12,21]$.

The equilibrium moments $\left\{n_{i}^{e q} \mid i=0,1, \ldots, 4\right\}$ corresponding to the distribution function $\left\{h_{i}^{e q} \mid i=0,1, \ldots, 4\right\}$ are:

$$
n_{0}^{e q}=r T, \quad n_{1}^{e q}=r u_{x} T, \quad n_{2}^{e q}=r u_{y} T, \quad n_{3}^{e q}=r \bar{\theta} T, \quad n_{4}^{e q}=0 .
$$

where $\mathrm{T}$ is the temperature and $\bar{\theta} \in(0,1)$ is a parameter. $\Psi$ is a source term given by $\Psi=\left(0,0, \alpha_{e} T \sigma_{2}, 0,0\right)^{T}$, and $\bar{H}$ is a correction term designed to eliminate the addition deviation term in the recovered macroscopic equation, which is given by $\bar{H}=\left(0, T F_{x}, T F_{y}, 0,0\right)^{T}$.

The temperature equilibrium distribution function $g_{i}^{e q}$ is given by: 


$$
g_{i}^{e q}=r T \varpi_{i}\left[1+\frac{e_{i} \cdot u}{c_{s T}{ }^{2}}\right]
$$

where $-\varpi_{0}=1 / 2$ and $\varpi_{i}=1 / 8$ for $i=1-4 . C_{s T}=\sqrt{\theta / 2}$ is the lattice sound speed of the $D 2 Q 5$ model.

The temperature $T$ and the effective thermal diffusivity $\alpha_{e}$ can be determined by:

$$
\begin{gathered}
T=\sum_{i=0}^{4} g_{i} \\
\alpha_{e}=c_{s T}{ }^{2}\left(\tau_{T}-0.5\right) \Delta T
\end{gathered}
$$

Where $\tau_{T}=\frac{1}{\sigma_{1}}=\frac{1}{\sigma_{2}}$.

\section{Results and discussion}

Evolution of heat transfers (Nusselt number) according to the porous medium for various numbers of Reynolds and Darcy is illustrate in fig. 2. We chose three cases presented for different Rayleigh numbers giving the three modes of predominant forced convection $\left(\mathrm{Ra}=10^{2}\right)$, mixed convection $\left(\mathrm{Ra}=10^{4}\right)$ and predominant natural convection $\left(\mathrm{Ra}=10^{6}\right)$. We note that for the three convection modes and for a high Darcy number $\left(\mathrm{Da}=10^{-2}\right)$ the influence of the porous medium is negligible characterized by horizontal steps following the increase in the porous medium height. Therefore, the reduction of the Darcy number increases the heat transfers but not in a very significant way, in our case (i.e., a maximum of approximately $6 \%$ for the porous medium height case: $\mathrm{H}=0.8$ compared to the totally fluid case). Also, when the Rayleigh number increases $\left(\mathrm{Ra}=10^{6}\right)$ fig. 8-c, note the independence of the transfers of the porous medium and thus of Darcy number and all the curves join, this is certainly due to the fact that the porous medium is far from the active wall and has very little influence on it. 

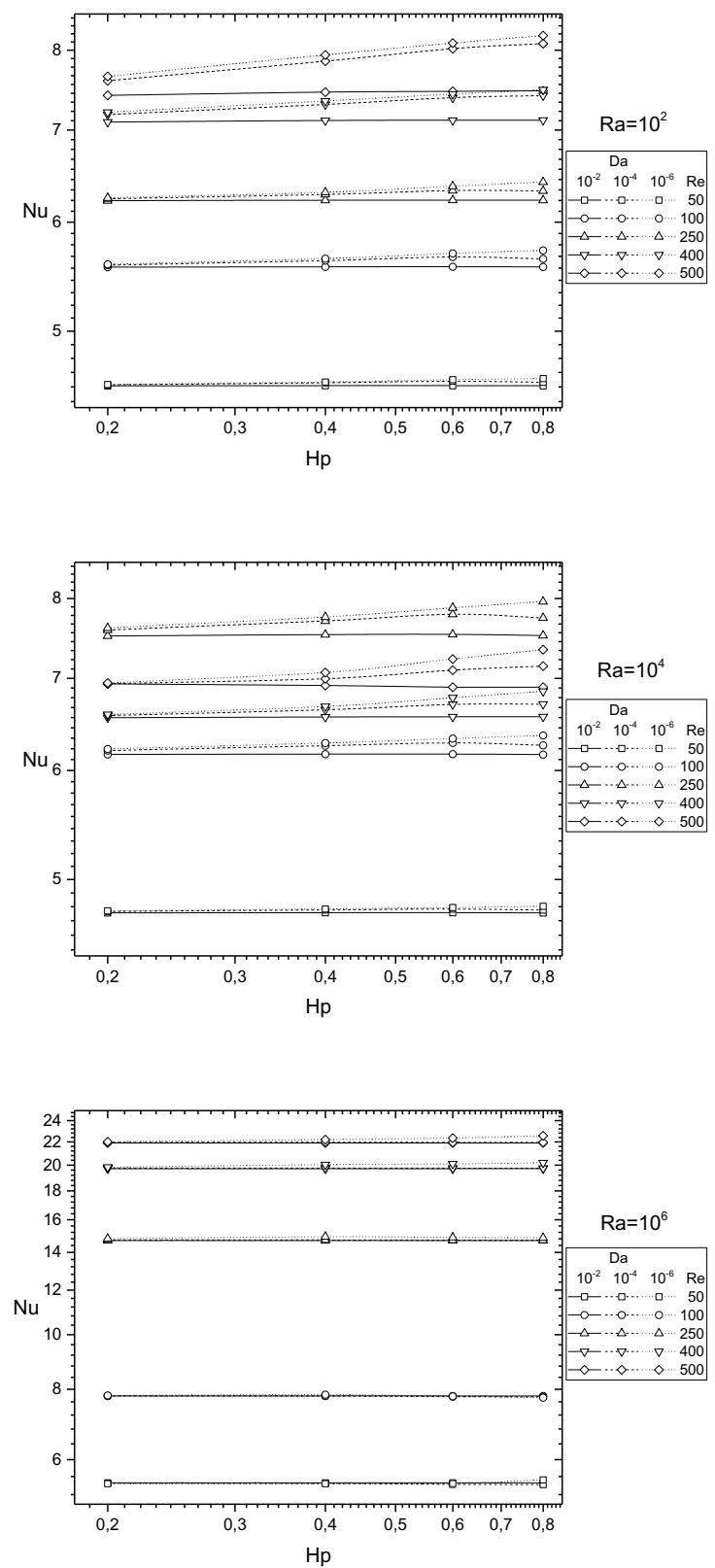

Fig. 2.Variation of $\mathrm{Nu}$ as a function of the height of the porous medium $\mathrm{H}_{\mathrm{p}}$ for different $\mathrm{Da}$ and for the case (a): $\mathrm{Ra}=10^{2}$, cases (b): $\mathrm{Ra}=10^{4}$ and case (c): $\mathrm{Ra}=10^{6}$

Average rate of heat transfer around the hot wall is calculated for different heights of porous partition is represented Fig. 3 and Fig.4. It is clear that the porous medium height has very little influence on the heat transfer curves. We also note that the two particular trends observed in mixed convection studies are found in our work. A constant level lines synonymous with predominant forced convection and oblique lines synonymous with predominant natural convection. The junction between the last two trends is considered as 
mixed convection. It should be mentioned that the dominant forced convection ends for Richardson numbers of $\mathrm{Ri}<0.5633$ while natural convection begins with Richardson $\mathrm{Ri}>5.633$.

The analysis of the dominant forced convection gives us evidence: The heat transfer rises with the increase in the Reynolds number. Indeed, the increase in the Reynolds number projects the fluid towards the heated wall (see the graphs of the stream lines function) thus increasing the flow passing the heated wall and subsequently causing a growth in the extracted heat (and therefore the Nusselt number).

The second trend (i.e. mixed convection and natural convection) gives interesting observations. The natural convection regime is never reached for low Reynolds numbers (i.e. $\mathrm{Re}=50$ and $\mathrm{Re}=100$ ). For those Reynolds, the cooperating natural convection improves transfers, only by around 10\%; while, for the other Reynolds the improvement in transfers due to natural and forced convection cooperation is significant.

It can be seen from analysis of mixed convection that the case $\mathrm{Ra}=10^{4}$ and $\mathrm{Re}=250$ give the best transfer rate compared to a larger Reynolds number. In this case the analysis of the stream functions reveals that a large part of the fluid entering the cavity is projected towards the wall increasing the parietal flow.

The augmentation in the porous medium height does not really change the evolution of the Nusselt number figures $\mathrm{Nu}=\mathrm{f}(\mathrm{Ra}, \mathrm{Re})$. The differences between these figures for different porous medium heights are reduced and it can be predicted that the porous medium height has little influence on the heat transfers and the most significant increase is obtained for a porous medium height $\mathrm{H}=0.8$, a Darcy number of $10-6$ where, we obtain an intensification of around $6 \%$ compared to the totally fluid case. 

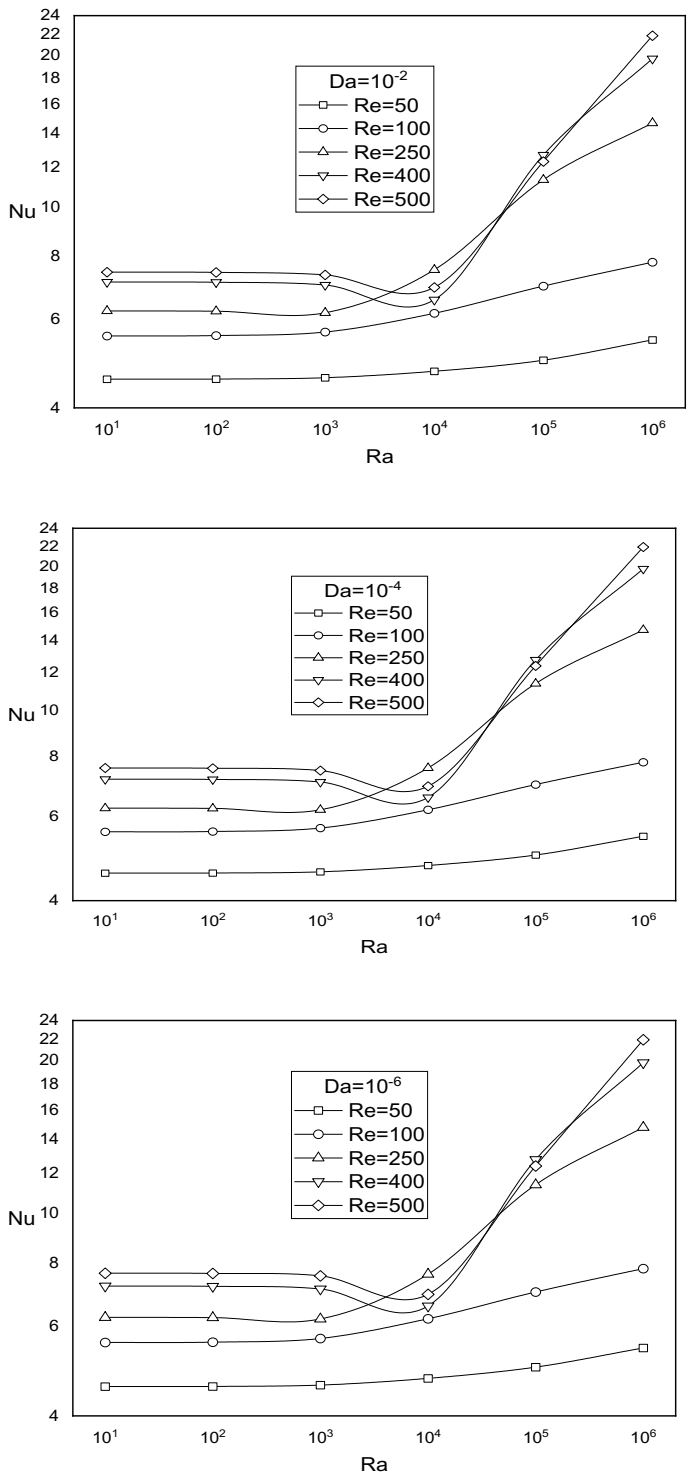

Fig 3.Evolution of heat transfer (Nusselt number) as a function of $\mathrm{Ra}$ for different $\mathrm{Re}, \mathrm{Da}$ and $\mathrm{H}_{\mathrm{p}}=0.4$ 

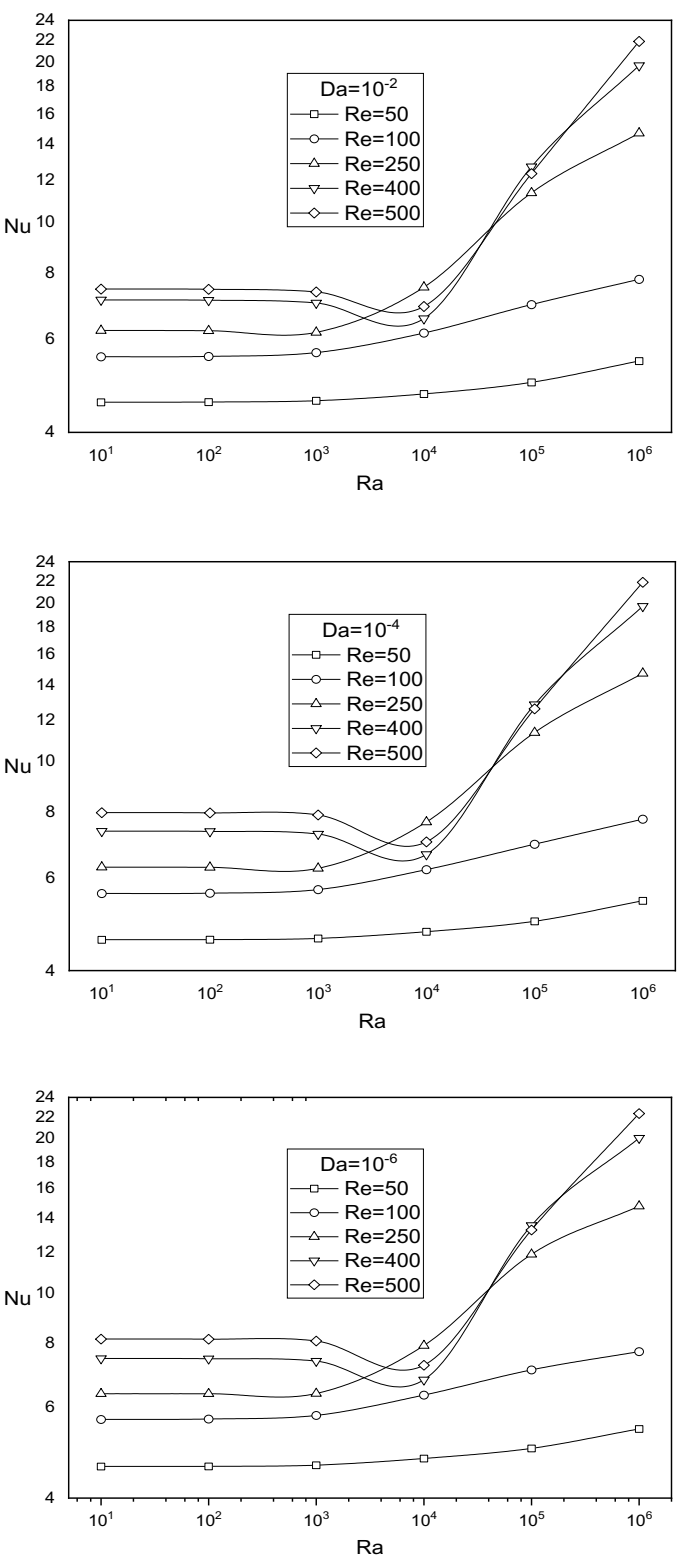

Fig 4. Evolution of heat transfer (Nusselt number) as a function of Ra for different $\mathrm{Re}, \mathrm{Da}$ and $\mathrm{Hp}=0.8$

\section{Conclusions}

The Lattice Boltzmann Multiple Relaxation Time method was undertaken to study numerically heat transfer in a ventilated cavity with an aspect ratio equal to two $(\mathrm{L} / \mathrm{H}=2)$ and a porous partition in the middle. The equation based on a generalized Darcy model extended to Brinkman-Forchheimer was combined to consider the flow in the porous medium.

The analysis of the heat transfer in the dominant forced convection case gives us evidence: The heat transfers increase with the increase of the Reynolds number values. The natural 
convection regime is never reached for low Reynolds numbers (i.e. $\operatorname{Re}=50$ and $\operatorname{Re}=100$ ). For these Re values, the cooperating natural convection only improves transfers by around $10 \%$ while, for the others Reynolds numbers the improvement in transfers due to natural and forced convections cooperation is significant. The elevation of the porous medium height does not really change the curves evolution in figures of the Nusselt number according to Ra and Re, Num=f(Ra, Re).

\section{References}

1. T. Gil-Lopez, et al., Energy and Buildings. 140, 371 (2017)

2. N.M. Mateus and Carrilho da Graça G., Building and Environment. 114, 470 (2017)

3. M. Krajčík, et al., Science and Technology for the Built Environment. 22, 317 (2015)

4. B. Rahmati, Heidarian A., and Jadidi A.M., Applied Thermal Engineering. 138, 861 (2018)

5. W. Shan and Rim D., Energy and Buildings. 158, 466 (2018)

6. N.M. Mateus and GraçaG.C.d., Energy and Buildings. 108, 44 (2015)

7. R. Kosonen, et al., Building and Environment. 108, 207 (2016)

8. B.E. Yuce and Pulat E., International Communications in Heat and Mass Transfer. 92, $1(2018)$

9. W. Liu, et al., Numerical Heat Transfer, Part A: Applications. 69, 748 (2016)

10. N.A. Rodriguez, Hinojosa J.F., and Xaman J., J. Heat Transf.-Trans. ASME. 137, 5 (2015)

11. L. Nasseri, et al., Numerical Heat Transfer, Part A: Applications. 75, 674 (2019)

12. L. Nasseri, et al., Eur. Phys. J. Appl. Phys. 86, 20902 (2019)

13. X. Shan, et al., Building and Environment. 102, 128 (2016)

14. W. Qiu-Wang and Zhen Z., Engineering Computations. 23, 218 (2006)

15. X.-H. Ren, et al., Applied Thermal Engineering. 94, 159 (2016)

16. X.-H. Ren, et al., Energy and Buildings. 138, 280 (2017)

17. C.T. Hsu and Cheng P., International Journal of Heat and Mass Transfer. 33, 1587 (1990)

18. T. Seta, Takegoshi E., and Okui K., Mathematics and Computers in Simulation. 72, 195 (2006)

19. S. Hassan, et al., Journal of Computational Engineering and Physical Modeling. 2, 21 (2019)

20. S. Ergun, Chem. Eng. Prog. 48, 89 (1952)

21. Q. Liu, et al., International Journal of Heat and Mass Transfer. 102, 1334 (2016)

22. F. Rong, et al., International Journal of Heat and Mass Transfer. 53, 5519 (2010)

23. Y. Guo, et al., International journal of Numerical methods for Heat \& fluid flow. (2010)

24. S. Khali, et al., Physical Review E. 87, 053002 (2013)

25. H. Dixit and Babu V., International journal of heat and mass transfer. 49, 727 (2006)

26. Q. Li, et al., International Journal of Modern Physics C. 19, 125 (2008)

27. M. Hortmann, Perić M., and Scheuerer G., International journal for numerical methods in fluids. 11, 189 (1990) 
28. Q. Liu, et al., International Journal of Heat and Mass transfer. 73, 761 (2014)

29. P. Nithiarasu, Seetharamu K., and Sundararajan T., International Journal of Heat and Mass Transfer. 40, 3955 (1997) 